\title{
Time variable cosmological constant of holographic origin with interaction in Brans-Dicke theory
}

\author{
Jianbo Lu, ${ }^{1, *}$ Lina Ma, ${ }^{1}$ Molin Liu, ${ }^{2}$ and Yabo $\mathrm{Wu}^{1}$ \\ ${ }^{1}$ Department of Physics, Liaoning Normal University, Dalian 116029, P. R. China \\ ${ }^{2}$ College of Physics and Electronic Engineering, Xinyang Normal University, Xinyang, 464000, P. R. China
}

Time variable cosmological constant (TVCC) of holographic origin with interaction in BransDicke theory is discussed in this paper. We investigate some characters for this model, and show the evolutions of deceleration parameter and equation of state (EOS) for dark energy. It is shown that in this scenario an accelerating universe can be obtained and the evolution of EOS for dark energy can cross over the boundary of phantom divide. In addition, a geometrical diagnostic method, jerk parameter is applied to this model to distinguish it with cosmological constant.

PACS numbers: 98.80.-k

Keywords: Time variable cosmological constant (TVCC); interaction; Brans-Dicke theory; geometrical diagnostic.

\section{Introduction}

The astronomical observations [1, 2] support that the universe is undergoing accelerated expansion. It indicates that there exist a new component dubbed as dark energy with negative pressure in universe. A natural explanation on the accelerated expansion is due to a positive tiny cosmological constant of holographic origin. Though the cosmological constant suffers the so-called fine tuning and cosmic coincidence problems, it fits the observations very well in $2 \sigma$ confidence level [3, 4]. However, if the cosmological constant is not a constant but a time variable one [5-17] or there is an interaction between dark matter and dark energy [18 29], the fine tuning and cosmic coincidence problems can be removed. In particular, the dynamic vacuum energy density based on holographic principle is investigated extensively 30 34]. According to the holographic principle, the total energy of a system with size $L$ should not exceed the mass of a black hole with the same size as the system, and the largest $L$ allows a energy density $\rho_{\Lambda}=3 c^{2} M_{p}^{2} L^{-2}$, where $c$ is a numerical constant and $M_{p}$ is the reduced Planck Mass $M_{p}^{-2}=8 \pi G$. It just means a duality between UV cut-off and IR cut-off which are related to the vacuum energy and the large scale of the universe, respectively. For example, Refs. [35, 36] have applied this principle to discuss Hubble horizon, future event horizon and particle horizon in cosmology. Another dark energy model with relations with holographic dark energy, named agegraphic dark energy, was also extensively researched [37-42]. This model is based on the well-known Heisenberg uncertainty relation to the universe. Therefore, the energy density of metric fluctuations in Minkowski space-time is $\rho_{\Lambda} \sim M_{p}^{2} / t^{2}$, where $t$ is time or length scale. Obviously, it looks like the holographic one, and there are some relations between them [37].

*Electronic address: lvjianbo819@163.com 
In addition, it is known that the de Sitter spacetime is described by the line element,

$$
d s^{2}=\left(1-\frac{r^{2}}{l^{2}}\right) d t^{2}+\left(1-\frac{r^{2}}{l^{2}}\right)^{-1} d r^{2}+r^{2}\left(d \theta^{2}+\sin ^{2} \theta d \varphi^{2}\right),
$$

where the cosmological constant is taken as $\Lambda=3 / l^{2}$. According to the horizon equation, cosmological horizon is obtained,

$$
r_{\Lambda}=c^{\prime} t_{\Lambda}=\sqrt{3 /|\Lambda|}
$$

where $c^{\prime}$ is the speed of light taken to unit here. Conversely, any cosmological length scale or time scale can introduce a energy density $\Lambda(t)$ into Einstein's theory

$$
\Lambda(t)=\frac{3}{r_{\Lambda}^{2}(t)}=\frac{3}{t_{\Lambda}^{2}(t)} .
$$

When a dynamic time scale is taken into account, a time variable cosmological constant (TVCC) is obtained. And a natural time scale is the age of our universe, $t_{\Lambda}=\int_{0}^{t} d t^{\prime}=\int_{0}^{a} \frac{d a^{\prime}}{a^{\prime} H}$. If ones take this cosmological horizon as the length scale (or time scale) of cut-off in de Sitter universe, vacuum energy density is expressed, $\rho_{\Lambda}=3 c^{2} M_{p}^{2} / t_{\Lambda}^{2}[43$. From this point, , one can see that this time variable cosmological constant is similar with the holographic and the agegraphic dark energy.

On the other hand, Brans-Dicke theory (BD) 44] as a natural extension to Einstein's gravitational theory of general relativity (GR), can pass the experimental tests from the solar system [45]. In Brans-Dicke theory, the gravitational "constant" $G$ is replaced with the inverse of a time-dependent scalar field $\Phi(t)$, which couples to gravity with a coupling parameter $\omega$. The holographic dark energy model in the framework of Brans-Dicke theory has already been considered by many people [46 50]. Here we study some characters of time variable cosmological constant in BD theory.

\section{Interacting time variable cosmological constant in Brans-Dicke theory}

In the framework of BD theory, the field is described by the action below,

$$
S=d^{4} x \sqrt{-g}\left[\Phi R-\frac{\omega}{\Phi} \Phi^{, \alpha} \Phi_{, \alpha}+L_{m}\right]
$$

where $L_{m}$ is the matter Lagrangian, $\Phi$ is the non-minimally coupled BD scalar field which plays the role of gravitational "constant" $G$ with relating to the inverse of the $\Phi$. The BD coupling parameter $\omega$ is restricted to be around 40000 according to the local gravity tests in the solar system [51]. Some proposals address that when the system changes from local to large cosmological scales $\omega$ should have more smaller value [52 54]. By varying above action with respect to the metric, the gravitational field equations is given as

$$
G_{\mu \nu}=\frac{1}{\Phi} T_{\mu \nu}^{m}+\frac{\omega}{\Phi^{2}}\left[\Phi_{, \mu} \Phi_{, \nu}-\frac{1}{2} g_{\mu \nu} \Phi_{, \alpha} \Phi^{, \alpha}\right]+\frac{1}{\Phi}\left[\Phi_{, \mu ; \nu}-g_{\mu \nu} \frac{T}{3+2 \omega}\right],
$$

where $G_{\mu \nu}$ is Einstein tensor, $T_{\mu \nu}^{m}$ denotes the energy-momentum tensor of matter, and $T=T_{\mu \nu}^{m} g^{\mu \nu}$. If we consider the detailed components of universe including the baryon matter $\rho_{b}$, the cold dark matter $\rho_{c}$, the radiation $\rho_{r}$, and the time variable cosmological constant $\rho_{\Lambda}$ as dark energy, the gravitational equations are written as

$$
3 \Phi\left[H^{2}+H \frac{\dot{\Phi}}{\Phi}-\frac{\omega}{6} \frac{\dot{\Phi}^{2}}{\Phi^{2}}\right]=\rho,
$$




$$
2 \frac{\ddot{a}}{a}+H^{2}+\frac{\omega}{2} \frac{\dot{\Phi}^{2}}{\Phi^{2}}+2 H \frac{\dot{\Phi}}{\Phi}+\frac{\ddot{\Phi}}{\Phi}=-\frac{p_{\Lambda}}{\Phi},
$$

where $H=\frac{\dot{a}}{a}$ is the Hubble parameter, $\rho=\rho_{b}+\rho_{c}+\rho_{r}+\rho_{\Lambda}, p_{\Lambda}$ is the pressure of TVCC, and its energy density is

$$
\rho_{\Lambda}=3 c^{2} \Phi(t) / t_{\Lambda}^{2}
$$

with time scale

$$
t_{\Lambda}=\int_{0}^{t} d t^{\prime}=\int_{0}^{a} \frac{d a^{\prime}}{a^{\prime} H^{\prime}}
$$

Submitting Eq. (9) into (8) and taking the derivative with respect to $\ln a$, we get

$$
\rho_{\Lambda}^{\prime}=\frac{d \rho_{\Lambda}}{d \ln a}=-\frac{6}{c} \Phi H^{2} \Omega_{\Lambda}^{\frac{3}{2}}+3 \Phi^{\prime} H^{2} \Omega_{\Lambda},
$$

with the definition of dimensionless energy density, $\Omega_{i}=\frac{\rho_{i}}{3 H^{2} \Phi}$, where $i$ can denote radiation $\Omega_{r}$, baryon matter $\Omega_{b}$, cold dark matter $\Omega_{c}$, and TVCC $\Omega_{\Lambda}$, respectively. We consider an interaction existed in the two dark components, the energy conservation equation of each component in universe is written respectively as,

$$
\begin{gathered}
\dot{\rho}_{r}+4 H \rho_{r}=0, \\
\dot{\rho}_{b}+3 H \rho_{b}=0, \\
\dot{\rho}_{c}+3 H \rho_{c}=Q, \\
\dot{\rho}_{\Lambda}+3 H\left(1+w_{\Lambda}\right) \rho_{\Lambda}=-Q .
\end{gathered}
$$

By adopting a generalized interacting term, $Q=\lambda_{1} H \rho_{c}+\lambda_{2} H \rho_{\Lambda}$, Eq.(14) is rewritten in a form as

$$
\rho_{\Lambda}^{\prime}+3\left(1+w_{\Lambda}\right) \rho_{\Lambda}=-\left(\lambda_{1} \rho_{c}+\lambda_{2} \rho_{\Lambda}\right)
$$

Combining Eqs.(10) and (15), we obtain the equation of state (EOS) of this interacting time variable cosmological constant as,

$$
w_{\Lambda}=\frac{2}{3 c} \Omega_{\Lambda}^{\frac{1}{2}}-\frac{1}{3} \frac{\Phi^{\prime}}{\Phi}-\frac{\lambda_{1} \Omega_{c}}{3 \Omega_{\Lambda}}-\frac{\lambda_{2}}{3}-1 .
$$

With considering a parameterized form for scalar field $\Phi=\Phi_{0}\left(\frac{a}{a_{0}}\right)^{\alpha}$, Friedmann equation (6) is rewritten as

$$
H^{2}=\frac{2 \rho}{\left(6+6 \alpha-\omega \alpha^{2}\right) \Phi} \text {. }
$$

Using Eqs. (6), (7) and (17), we get

$$
\dot{H}=\frac{-3}{\left(6+6 \alpha-\omega \alpha^{2}\right) \Phi}\left[\left(\rho+p_{\Lambda}\right)+\frac{\alpha}{3} \rho\right] .
$$

Substituting Eq.(16) into (15) and applying the definition of $\Omega_{\Lambda}$, we obtain

$$
\frac{H^{\prime}}{H}=\frac{1}{2 \Omega_{\Lambda}}\left[-\Omega_{\Lambda}^{\prime}-\frac{2}{c} \Omega_{\Lambda}^{\frac{3}{2}}\right]
$$


On the other hand, substituting $\dot{H}=H^{\prime} H$ and $p_{\Lambda}=w_{\Lambda} \rho_{\Lambda}$ into Eq.(18), we have

$$
\frac{H^{\prime}}{H}=\frac{-9}{6+6 \alpha-\omega \alpha^{2}}\left[\Omega_{r}+\Omega_{m}+\frac{2}{3 c} \Omega_{\Lambda}^{\frac{3}{2}}-\frac{1}{3} \frac{\Phi^{\prime}}{\Phi} \Omega_{\Lambda}-\frac{\lambda_{1}}{3} \Omega_{c}-\frac{\lambda_{2}}{3} \Omega_{\Lambda}+\frac{\alpha}{3}\left(\Omega_{r}+\Omega_{m}+\Omega_{\Lambda}\right)\right],
$$

with $\Omega_{m}=\Omega_{b}+\Omega_{c}$. Thus, combining Eqs.(19) and (20) we get the differential equation for $\Omega_{\Lambda}$,

$$
\frac{\Omega_{\Lambda}^{\prime}}{\Omega_{\Lambda}}=\frac{18}{6+6 \alpha-\omega \alpha^{2}}\left[\Omega_{r}+\Omega_{m}+\frac{2}{3 c} \Omega_{\Lambda}^{\frac{3}{2}}-\frac{1}{3} \frac{\Phi^{\prime}}{\Phi} \Omega_{\Lambda}-\frac{\lambda_{1}}{3} \Omega_{c}-\frac{\lambda_{2}}{3} \Omega_{\Lambda}+\frac{\alpha}{3}\left(\Omega_{r}+\Omega_{m}+\Omega_{\Lambda}\right)\right]-\frac{2}{c} \Omega_{\Lambda}^{\frac{1}{2}} .
$$

In addition, using $\Phi=\Phi_{0}\left(\frac{a}{a_{0}}\right)^{\alpha}, \Phi^{\prime}=\frac{d \Phi}{d \ln a}=\alpha \Phi$, and $\Omega_{r}+\Omega_{m}+\Omega_{\Lambda}=1$, the derivative expressions of Hubble parameter and energy density for TVCC as a function of redshift $z$, can be expressed,

$$
\begin{gathered}
\frac{d H}{d z}=-\frac{H}{1+z} \frac{-9}{6+6 \alpha-\omega \alpha^{2}}\left[\Omega_{r}+\Omega_{m}+\frac{2}{3 c} \Omega_{\Lambda}^{\frac{3}{2}}-\frac{\alpha}{3} \Omega_{\Lambda}-\frac{\lambda_{1}}{3} \Omega_{c}-\frac{\lambda_{2}}{3} \Omega_{\Lambda}+\frac{\alpha}{3}\right], \\
\frac{d \Omega_{\Lambda}}{d z}=-\frac{\Omega_{\Lambda}}{1+z}\left[\frac{18}{6+6 \alpha-\omega \alpha^{2}}\left(\Omega_{r}+\Omega_{m}+\frac{2}{3 c} \Omega_{\Lambda}^{\frac{3}{2}}-\frac{\alpha}{3} \Omega_{\Lambda}-\frac{\lambda_{1}}{3} \Omega_{c}-\frac{\lambda_{2}}{3} \Omega_{\Lambda}+\frac{\alpha}{3}\right)-\frac{2}{c} \Omega_{\Lambda}^{\frac{1}{2}}\right] .
\end{gathered}
$$

And the deceleration parameter $q$ is derived,

$$
q=-\frac{\ddot{a}}{a H^{2}}=\frac{2}{2+\alpha}\left[\frac{1}{2}+\Omega_{\Lambda}\left(\frac{1}{c} \Omega_{\Lambda}^{\frac{1}{2}}-\frac{1}{2} \alpha-\frac{1}{2} \lambda_{1} \frac{\Omega_{c}}{\Omega_{\Lambda}}-\frac{1}{2} \lambda_{2}-\frac{3}{2}\right)+\alpha\left(\frac{\omega}{4} \alpha+\frac{1}{2} \alpha+\frac{1}{2}\right)\right] .
$$

In above equations, for $\lambda_{1}=\lambda_{2}=0$, they reduce to the non-interacting cases; for $\omega \rightarrow \infty$ and $\alpha=0$, they correspond to the time variable cosmological constant in GR theory.
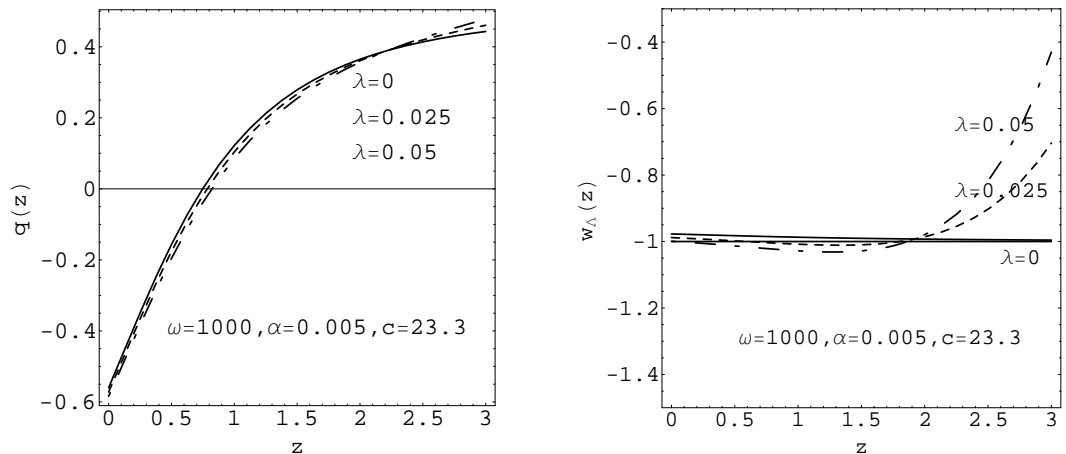

FIG. 1: The evolutions of EOS $w_{\Lambda}(z)$ and decelerating parameter $q(z)$ for interacting time variable cosmological constant in Brans-Dicke theory.

According to the Eqs.(16) and (24), the evolutions of $\operatorname{EOS} w_{\Lambda}(z)$ and decelerating parameter $q(z)$ for interacting time variable cosmological constant in Brans-Dicke theory are illustrated in Fig. 1, with the values of model parameters $\omega=1000[52], \alpha=0.005[55], c=23.3[56]$, where the three lines correspond respectively to the cases of parameter $\lambda=0, \lambda=0.025, \lambda=0.05$ for interacting case $Q=\lambda H\left(\rho_{c}+\rho_{\Lambda}\right)$. According to the Fig. 1, it can be seen that an expansion of universe from deceleration to acceleration is given in this model. And one can see that the shape of deceleration parameter $q(z)$ is not sensitive to the values of interaction parameter $\lambda$. For the evolution of EOS $w_{\Lambda}(z)$, at low redshift it is similar to cosmological constant, and at high redshift its evolution is dependent on the values of interaction parameter. In addition, in this scenario it is shown that the evolution of EOS $w_{\Lambda}(z)$ can cross over the boundary of phantom divide [57], as realized in the quintom model [8]. For the case of $\lambda=0.025$, the values of 
transition redshift, current deceleration parameter and EOS are given respectively as $z_{T}=0.784, q_{0}=-0.572$, and $w_{0 \Lambda}=-0.988$, which are consistent with the current observational constraint, where the model independent scenarios are constrained from the latest observational data [58].

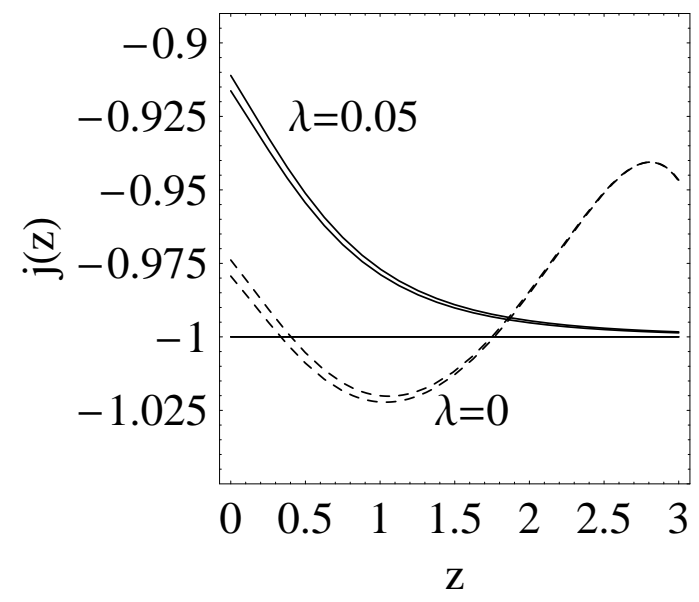

FIG. 2: The evolutions of $j(z)$ for time variable cosmological constant in Brans-Dicke theory and in general relativity, respectively.

Next we use a geometrical diagnostic method, i.e. jerk parameter, to distinguish the cosmological constant model and the interacting TVCC. Jerk parameter $j$ is defined as,

$$
j \equiv-\frac{1}{H^{3}}\left(\frac{\dot{a}}{a}\right)=-\left[\frac{1}{2}(1+z)^{2} \frac{\left[H(z)^{2}\right]^{\prime \prime}}{H(z)^{2}}-(1+z) \frac{\left[H(z)^{2}\right]^{\prime}}{H(z)^{2}}+1\right] .
$$

With the help of parameter $j$, the transitions between phases of different cosmic acceleration can be more conveniently described. And Eq. (25) can be derived as

$$
j=-1-\frac{9}{2} w_{d e} \Omega_{d e}\left(1+w_{d e}\right)+\frac{3}{2} \Omega_{d e} \frac{\dot{w}_{d e}}{H},
$$

where $\Omega_{d e}$ denotes dimensionless energy density for dark energy, and EOS for dark energy $w_{d e}$ can be either larger or smaller than -1. Also, from Eq. (26) it is easy to see that, for the cosmological constant model $\left(w_{\Lambda}(z)=-1\right)$, it has a constant jerk with $j(z)=-1$. Thus the jerk parameter can provide us with a convenient approach to search for departures from the cosmic concordance model, just as deviations from $w_{\Lambda}(z)=-1$ done in more standard dynamical analysis. According to Eq.(26), the evolutions of jerk parameter $j(z)$ are plotted in Fig. 2 for different cases, where the two dotted lines denote the cases of non-interacting time variable cosmological constant in BD and GR gravitational theory, respectively, as well as two solid lines denoting interacting cases. It is shown that the evolutions of $j(z)$ are similar in these two gravitational theories for both interacting and non-interacting TVCC. Also, in Fig. 2 one can see the departures from the cosmological constant for these four cases. 

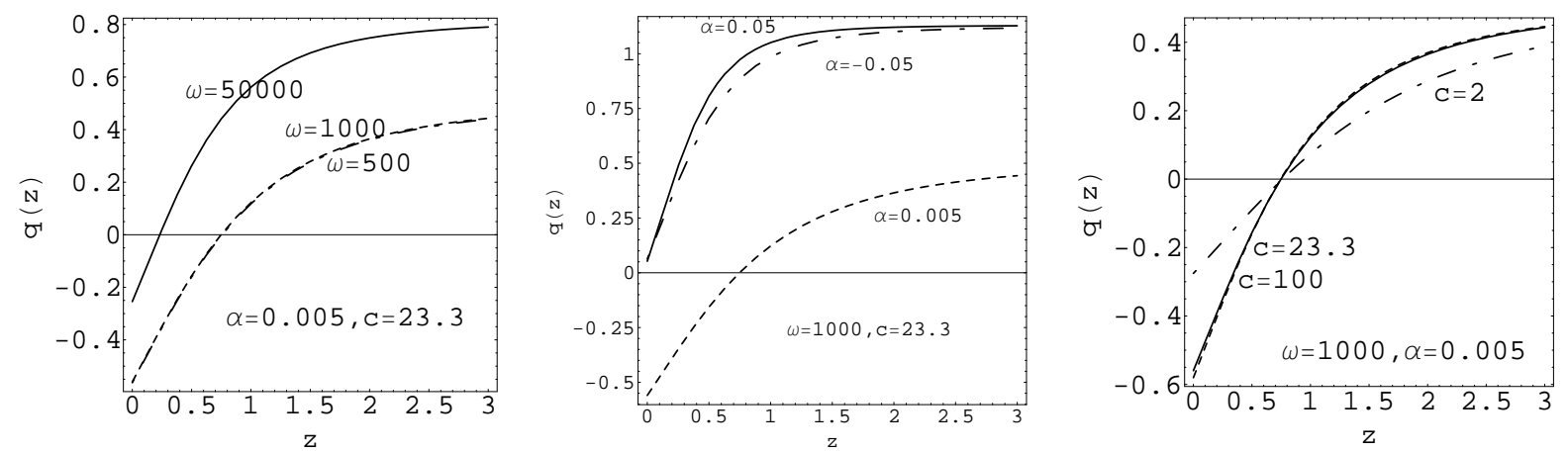

FIG. 3: The evolutions of $q(z)$ for time variable cosmological constant in Brans-Dicke theory with using various model parameters.
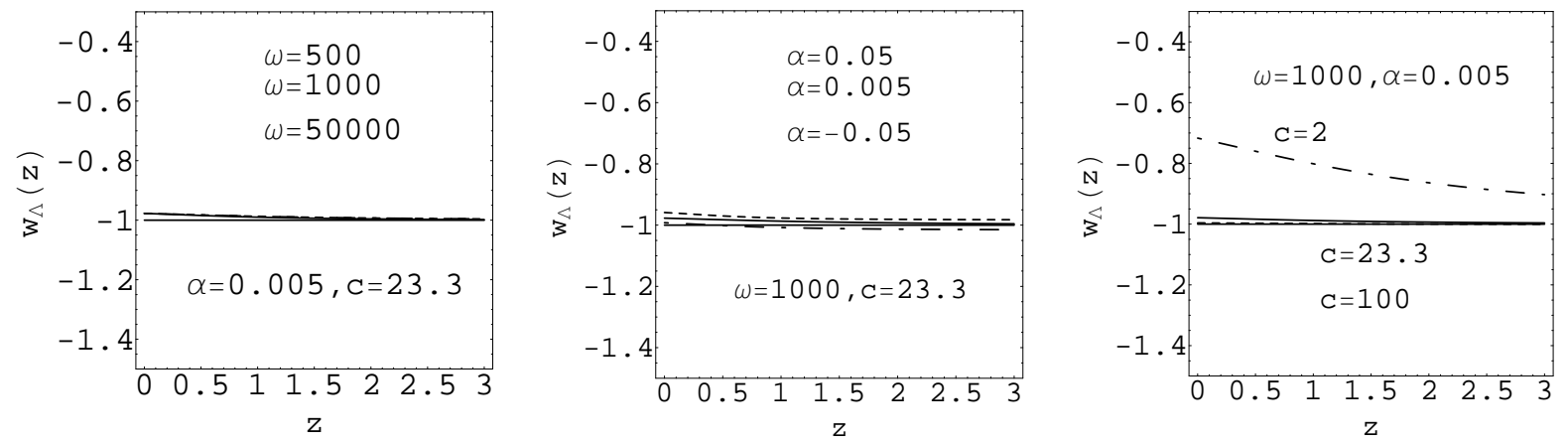

FIG. 4: The evolutions of $w_{\Lambda}(z)$ for time variable cosmological constant in Brans-Dicke theory with using various model parameters.

\section{Time variable cosmological constant in Brans-Dicke theory}

Next we investigate the evolutions of cosmological quantities for taking different values of model parameters to show their dependence on model parameters. We firstly consider the case of TVCC with no interaction in Brans-Dicke theory. Here the evolutions of deceleration parameter and EOS for TVCC model are plotted in Figs. 3 and 4 , with various parameters: (1) $\omega=(500,1000,50000), \alpha=0.005, \mathrm{c}=23.3 ;(2) \omega=1000, \alpha=(-0.05,0.005,0.05), \mathrm{c}=23.3 ;(3)$ $\omega=1000, \alpha=0.005, \mathrm{c}=(2,23.3,100)$. From the evolutions of $q(z)$ and $w_{\Lambda}(z)$ in Figs. 3 and 4 , one can see that $q(z)$ is more dependent on the values of $\alpha$ and $\omega$, but $w_{\Lambda}(\mathrm{z})$ is relative less dependent on them. And for the case of $\omega \rightarrow \infty, \alpha=0$ in the framework of GR theory, $q(z)$ and $w_{\Lambda}(\mathrm{z})$ are plotted in Fig. 5 with $\mathrm{c}=(2,23.3,100)$. Comparing this figure with Figs. 3 and 4, we can see that the differences of the evolutions of $q(z)$ and $w_{\Lambda}(z)$ between BD theory and GR theory are not obvious.

\section{Time variable cosmological constant with interaction}

In this part we consider different interactions for time variable cosmological constant in GR theory, i.e. taking $\omega \rightarrow \infty, \alpha=0$. For Figs. [6 and 7 the parameters are taken respectively as, (1) $c=23.3, \lambda=\lambda_{1}=\lambda_{2}=(0.05,0$, 

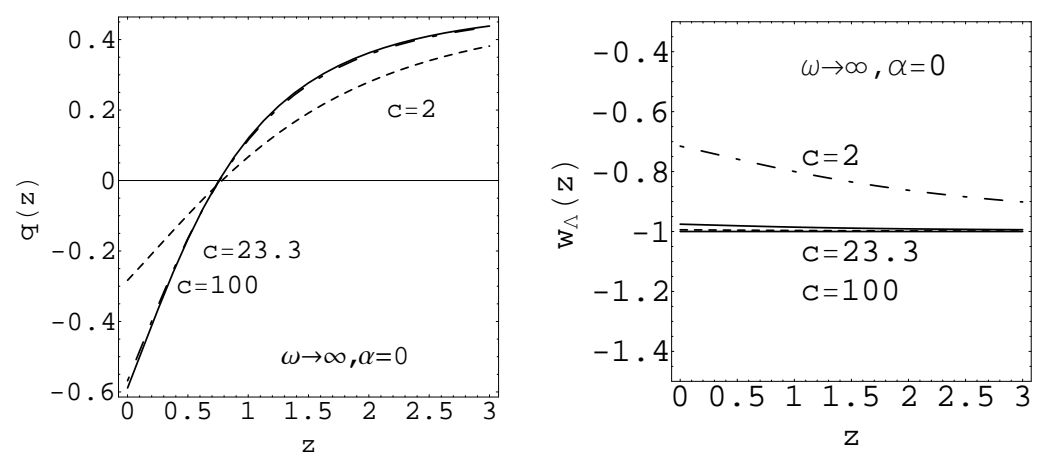

FIG. 5: The evolutions of $q(z)$ and $w_{\Lambda}(z)$ for time variable cosmological constant in GR theory with using different values of $c$.

$-0.05) ;(2) c=23.3, \lambda_{1}=(0.05,0,-0.05), \lambda_{2}=0 ;(3) c=23.3, \lambda_{1}=0, \lambda_{2}=(0.05,0,-0.05)$. According to the Figs. [6 and 7 one can see that, the shape of deceleration parameter $q(z)$ is not sensitive to the values of interaction parameter and its interacting forms; for the evolution of $w_{\Lambda}(z)$ it is not sensitive to the variable of parameter values $\lambda_{2}$, and they are similar for the cases of $Q=\lambda H\left(\rho_{c}+\rho_{\Lambda}\right)$ and $Q=\lambda_{1} H \rho_{c}$. And from Fig. 7 , it can be seen that the EOS can cross over the phantom boundary $w_{\Lambda}=-1$ for the exist of the interacting parameter $\lambda$ or $\lambda_{1}$. But for the case of negative interaction parameter $\lambda<0$ (or $\lambda_{1}<0$ ), it is found that there is a smaller value of EOS at higher redshift. In addition, in Fig. 8 we show the non-interacting case $\lambda=\lambda_{1}=\lambda_{2}=0$ with using $c=(-2,2,23.3)$. According to Fig. 8. it is indicated that for using the parameter value $c \in(2, \infty)$ and $c \in(-\infty,-2)$, the current value of $w_{0 \Lambda}$ lies in $(-1.285,-0.715)$, and the value of current deceleration parameter $q_{0} \in(-0.907,-0.283)$, transition redshift $z_{T} \in(0.681$, $0.782)$.
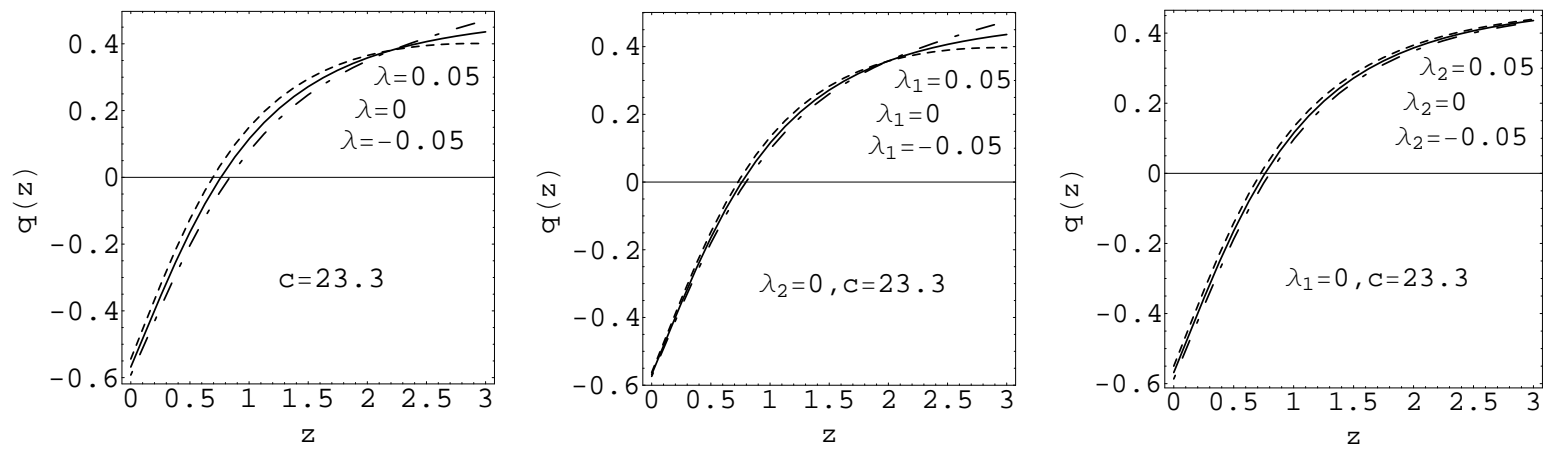

FIG. 6: The evolutions of $q(z)$ for interacting time variable cosmological constant.

\section{Conclusions}

In this paper the time variable cosmological constant with interaction in Brans-Dicke theory is discussed. One can see that an accelerating universe at late time is obtained in this model. And the equation of state for the interacting case can cross over the phantom boundary. Furthermore, by taking the model parameters to be in large regions, $\omega \geq 500, \alpha \in[-0.05,0.05], \lambda \in[-0.05,0.05]$, we investigate the evolutions of cosmological quantities for time variable 

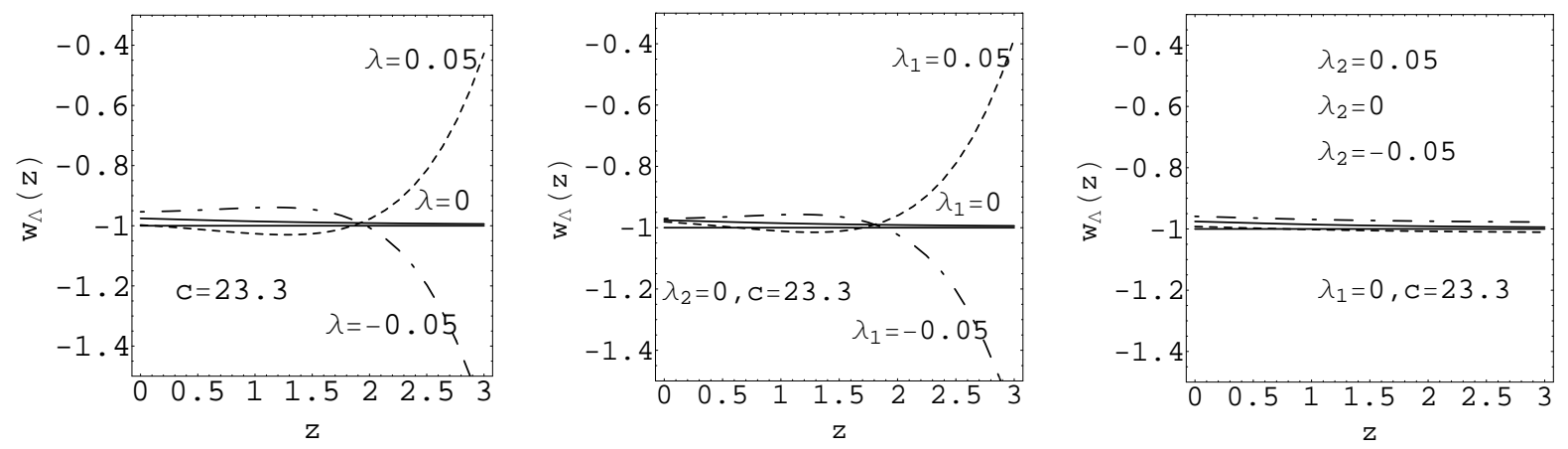

FIG. 7: The evolution of $w_{\Lambda}(z)$ for interacting time variable cosmological constant.
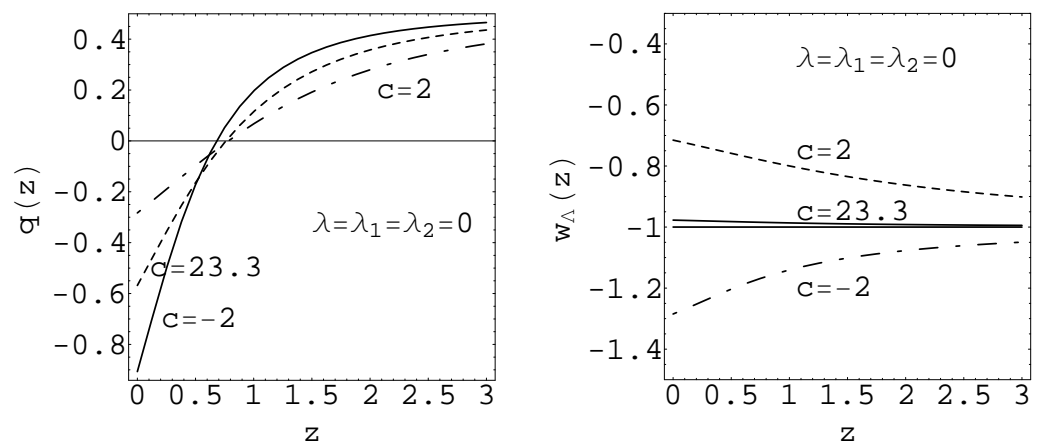

FIG. 8: The evolutions of $q(z)$ and $w_{\Lambda}(z)$ for non-interacting time variable cosmological constant.

cosmological constant. It is shown that the shape of deceleration parameter $q(z)$ is not sensitive to the values of interaction parameter and its interacting forms, but it is relatively more dependent on the values of BD parameter $\alpha$ and $\omega$. For the evolution of $\operatorname{EOS} w_{\Lambda}(z)$, it is more dependent on the values of interaction parameter, but is less sensitive to the variation of $\mathrm{BD}$ parameter $\alpha$ and $\omega$. In addition we can see that for the evolutions of deceleration parameter, EOS, and jerk parameter geometrical method, the differences between BD and GR theory are not obvious for time variable cosmological constant.

Acknowledgments The research work is supported by the National Natural Science Foundation (Grant No. 10875056), NSF (10703001) and NSF (No.11005088) of P.R. China.

\section{Appendix A: Investigate interaction between dark sections with a different interacting form $Q=\lambda_{3} \dot{\rho}_{\Lambda}$}

Also, for comparison we consider another interacting form $Q=\lambda_{3} \dot{\rho}_{\Lambda}$, which is consistent with the analysis of dimension according to the Eq. (14). Obviously, this interacting term is different from the above one $Q=\lambda_{1} H \rho_{c}+$ $\lambda_{2} H \rho_{\Lambda}$. For this case the corresponding expressions of some cosmological quantities in BD theory are derived as

$$
\begin{gathered}
w_{\Lambda}=\frac{2}{3 c} \Omega_{\Lambda}^{\frac{1}{2}}+\frac{2 \lambda_{3}}{3 c} \Omega_{\Lambda}^{\frac{1}{2}}-\frac{\alpha}{3}-\frac{\alpha \lambda_{3}}{3}-1, \\
\frac{d \Omega_{\Lambda}}{d z}=-\frac{\Omega_{\Lambda}}{1+z}\left[\frac{18}{6+6 \alpha-\omega \alpha^{2}}\left(\Omega_{r}+\Omega_{m}+\frac{2}{3 c} \Omega_{\Lambda}^{\frac{3}{2}}+\frac{2 \lambda_{3}}{3 c} \Omega_{\Lambda}^{\frac{3}{2}}-\frac{\alpha}{3} \Omega_{\Lambda}-\frac{\alpha \lambda_{3}}{3} \Omega_{\Lambda}+\frac{\alpha}{3}\right)-\frac{2}{c} \Omega_{\Lambda}^{\frac{1}{2}}-\frac{2 \lambda_{3}}{c} \Omega_{\Lambda}^{\frac{1}{2}}+\lambda_{3} \alpha-\lambda_{3}\right],
\end{gathered}
$$




$$
q=\frac{2}{2+\alpha}\left[\frac{1}{2}+\Omega_{\Lambda}\left(\frac{1}{c} \Omega_{\Lambda}^{\frac{1}{2}}+\frac{\lambda_{3}}{c} \Omega_{\Lambda}^{\frac{1}{2}}-\frac{1}{2} \alpha-\frac{\lambda_{3}}{2} \alpha-\frac{3}{2}\right)+\alpha\left(\frac{\omega}{4} \alpha+\frac{1}{2} \alpha+\frac{1}{2}\right)\right]
$$

with using a parameterized form for BD scalar field $\Phi=\Phi_{0}\left(\frac{a}{a_{0}}\right)^{\alpha}$. And the evolutions of deceleration parameter and EOS for this interacting time variable cosmological constant in the framework of BD theory are plotted in Fig 9 , From this figure we can see that, comparing with the non-interacting case the influences on the evolutions of $q(z)$ and $w_{\Lambda}(z)$ from this interacting form are not obvious, so we do not discuss much for this case in the text.
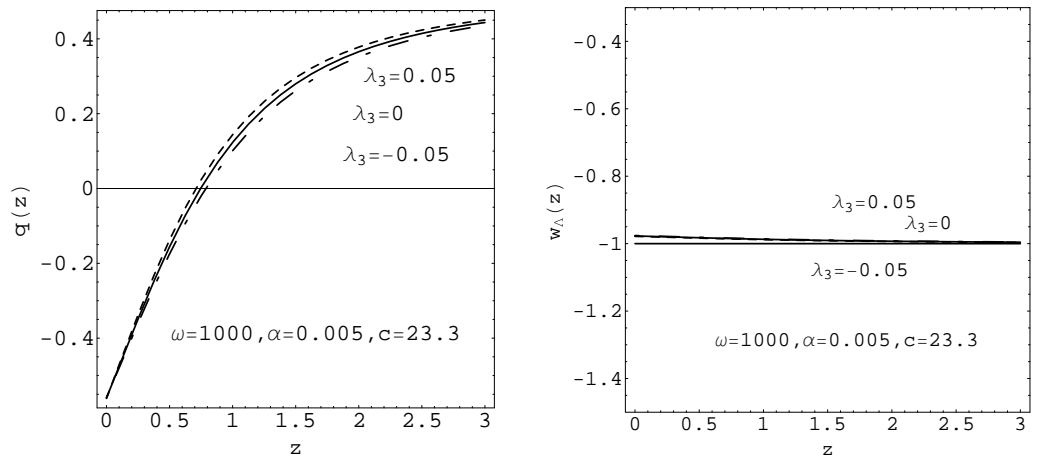

FIG. 9: The evolutions of $q(z)$ and $w_{\Lambda}(z)$ for interacting time variable cosmological constant in Brans-Dicke theory, with the interaction from $Q=\lambda_{3} \dot{\rho}_{\Lambda}$.

[1] A.G. Riess et al, 1998 Astron. J. 1161009 arXiv:astro-ph/9805201

[2] S. Perlmutter et al, 1999 Astrophys. J. 517, 565

[3] E. Komatsu, et al., Astrophys. J. Suppl. 180 (2009) 330, arXiv:0803.0547.

[4] J.B. Lu, L.X. Xu, and M.L. Liu, Phys.Lett.B 699, 246 (2011).

[5] J. Sola H. Stefancic, 2005 Phys.Lett.B 624,147-157 astro-ph/0505133

[6] M. Jamil, E.N. Saridakis, and M.R. Setare, 2009 Phys.Lett.B 679,172-176 [hep-th/0906.2847]

[7] I.L. Shapiroa, J. Sola, 2009 Phys.Lett.B 682,105-113 [hep-th/0910.4925].

[8] B. Feng, X.L. Wang and X.M. Zhang, 2005 Phys. Lett. B 607, 35 arXiv:astro-ph/0404224.

[9] B. Ratra and P.J.E. Peebels, 1988 Phys. Rev. D. 373406

[10] R.R. Caldwell, M. Kamionkowski and N. N. Weinberg, 2003 Phys. Rev. Lett. 91, 071301 arXiv:astro-ph/0302506

[11] L.X. Xu, J.B. Lu and W.B. Li, 2009 Eur. phys. J. C 64, 89

[12] J.B. Lu, E.N. Saridakis, M.R. Setare and L.X. Xu. JCAP, 2010, 03: 031 [arXiv:astro-ph/0912.0923]

[13] Y.T. Wang and L.X. Xu, Phys. Rev. D 81083523 (2010) arXiv:1004.3340

[14] J.B. Lu et al, Eur Phys J C 2011, 71:1800

[15] J.B. Lu, L.X. Xu, M.L. Liu and Y.X Gui, 2008 Eur. Phys. J. C 58311

[16] J.B. Lu, Phys. Lett. B 680, 404 (2009)

[17] J.B. Lu, Y.X. Gui and L.X. Xu, 2009 Eur. phys. J. C 63349.

[18] A. P. Billyard, A.A. Coley, 2000 Phys.Rev.D 61,083503 astro-ph/9908224

[19] X.M. Chen, Yungui Gong, E.N. Saridakis, 2009 JCAP 0904,001 [gr-qc/0812.1117].

[20] B. Wang, C.Y. Lin, E.o Abdalla, Phys. Lett. B 637 (2006) 357

[21] H. Kim, H.W. Lee, Y.S. Myung, Phys. Lett. B 632 (2006) 605 
[22] B. Hu, Y. Ling, Phys. Rev. D 73 (2006) 123510

[23] W. Zimdahl, D. Pavon, arXiv:astro-ph/0606555

[24] H.M. Sadjadi, JCAP 0702 (2007) 026

[25] Q. Wu, Y. Gong, A. Wang, J.S. Alcaniz, arXiv:0705.1006

[26] J.F. Zhang, X. Zhang, H.Y. Liu, Phys. Lett. B 659 (2008) 26

[27] C. Feng, B. Wang, Y. Gong, R.K. Su, arXiv:0706.4033

[28] S.F. Wu, P.M. Zhang, G.H. Yang, Class. Quantum Grav. 26 (2009) 055020

[29] M.A. Rashid, M.U. Farooq, M. Jamil, arXiv:0901.3724.

[30] M. Li, Phys. Lett. B 603 (2004) 1, hep-th/0403127

[31] Q.G. Huang, M. Li, JCAP 0503 (2005) 001, hep-th/0410095

[32] B. Chen, M. Li, Y. Wang, Nucl. Phys. B 774 (2007) 256, astro-ph/0611623

[33] J.F. Zhang, X. Zhang, H.Y. Liu, Eur. Phys. J. C 52 (2007) 693, arXiv:0708.3121

[34] S.D.H. Hsu, Phys. Lett. B 594 (2004) 13, arXiv:hep-th/0403052

[35] P. Horava, D. Minic, Phys. Rev. Lett. 85 (2000) 1610

[36] C.J. Feng, Phys. Lett. B 663 (2008) 367.

[37] R.G. Cai, Phys. Lett. B 657 (2007) 228, arXiv:0707.4049 [hep-th].

[38] H. Wei, R.G. Cai, Phys. Lett. B 660 (2008) 113, arXiv:0708.0884 [astro-ph]

[39] M. Maziashvili, Phys. Lett. B 652 (2007) 165, arXiv:0705.0924 [gr-qc]

[40] H. Wei, R.G. Cai, Phys. Lett. B 655 (2007) 1, arXiv:0707.4526 [gr-qc]

[41] X. Wu, Y. Zhang, H. Li, R.G. Cai, Z.H. Zhu, arXiv:0708.0349 [astro-ph]

[42] Y. Zhang, H. Li, X. Wu, H. Wei, R.G. Cai, arXiv:0708.1214][astro-ph].

[43] L.X. Xu, J.B. Lu, W.B. Li, Physics Letters B 690 (2010) 333-336.

[44] C.H. Brans, R.H. Dicke, Phys. Rev. 124, 925 (1961).

[45] B. Bertotti, L. Iess, P. Tortora, Nature 425, 374 (2003).

[46] L.X. Xu, W.B. Li, J.B. Lu, Eur. Phys. J. C 60 (2009) 135

[47] Y. Gong, Phys. Rev. D 70, 064029 (2004). hep-th/0404030

[48] M.R. Setare, Phys. Lett. B 644, 99 (2007). hep-th/0610190

[49] N. Banerjee, D. Pavon, Phys. Lett. B 647, 447 (2007)

[50] J.B. Lu, L.X. Xu, Y.B. Wu, M.L. Liu, Gen.Rel.Grav.43:819,2011.

[51] C. M. Will, Living Rev. Rel. 9, 3 (2005).

[52] V. Acquaviva, L. Verde, JCAP 12, 001 (2007), arXiv:0709.0082.

[53] J.C. Fabris, S.V.B. Goncalves, R. Ribeiro, Grav.Cosmol.12:49-54,2006, arXiv:astro-ph/0510779

[54] P.D. Mannheim, Found. of Phys. 30, 709 (2000).

[55] J.B. Lu, W.P. Wang, L.X. Xu, Y.B. Wu, Eur. Phys. J. Plus (2011) 126: 92, arXiv:1105.1868.

[56] Y. Chen, Z.H. Zhu, L.X. Xu, J. S. Alcaniz, Phys. Lett. B, 698:175-182,2011. arXiv:1103.2512.

[57] Y.F. Cai, E.N. Saridakis b, M.R. Setare, and J.Q. Xia, Physics Reports, 493, 1-60 (2010) arXiv:0909.2776].

[58] J.B. Lu, L.X. Xu, Y.B. Wu, Modern Physics Letters A, 25 (2010) 3033-3046. 